INPLASY

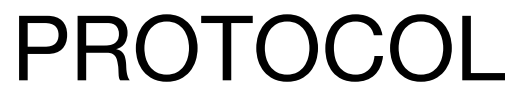

To cite: Wu et al. Diagnostic accuracy of contrastenhanced ultrasound in the detection of small renal masses: A protocol of systematic review and metaanalysis. Inplasy protocol 202060040. doi: 10.37766/inplasy2020.6.0040

Received: 11 June 2020

Published: 11 June 2020

Corresponding author: Jiangfeng Wu

wjfhospital@163.com

Author Affiliation:

Dongyang People's Hospital

Support: Jinhua Sci. \& Tech Bureau.

Review Stage at time of this submission: The review has not yet started.

Conflicts of interest: None.

\section{Diagnostic accuracy of contrast- enhanced ultrasound in the detection of small renal masses: A protocol of systematic review and meta-analysis}

Wu, JF'; Wu, C2; Wang, YL³; Wang, ZP4.

Review question / Objective: The diagnostic accuracy of contrast-enhanced ultrasound (CEUS) for small renal masses (SRMs) are apparently variable among previous studies. Hence, this study will evaluate the diagnostic accuracy of CEUS in the identification of benign and malignant SRMs.

Condition being studied: Contrast-enhanced ultrasound; small renal masses; histopathology.

Information sources: Cochrane Library, EMBASE, Pubmed, WANGFANG and China National Knowledge Infrastructure will be systematically searched to identify potentially eligible studies from inception to May 2020. Computer searches will be carried out using the Medical Subject Heading and keywords. There will be no limitations of language and publication status. The bibliographies of identified studies and review Articles will be manually screened to expand the number of eligible studies.

INPLASY registration number: This protocol was registered with the International Platform of Registered Systematic Review and Meta-Analysis Protocols (INPLASY) on 11 June 2020 and was last updated on 11 June 2020 (registration number INPLASY202060040).

\section{INTRODUCTION}

Review question / Objective: The diagnostic accuracy of contrast-enhanced ultrasound (CEUS) for small renal masses (SRMs) are apparently variable among previous studies. Hence, this study will evaluate the diagnostic accuracy of CEUS in the identification of benign and malignant SRMs.

Condition being studied: Contrastenhanced ultrasound; small renal masses; histopathology. 


\section{METHODS}

Participant or population: Patients with small renal masses.

Intervention: CEUS for the diagnosis of SRMs will be included.

Comparator: Histopathology, cytopathology, and/or clinical follow-up.

Study designs to be included: Randomised control trials, case control studies or prospective studies.

Eligibility criteria: 1. Randomised control trials, case control studies or prospective studies will be included. 2. Studies involving patients with small renal masses will be included. 3. Studies using CEUS for the diagnosis of SRMs will be included. 4.Studies using reference standards such as histopathology, cytopathology, and/or clinical follow-up will be included.

Information sources: Cochrane Library, EMBASE, Pubmed, WANGFANG and China National Knowledge Infrastructure will be systematically searched to identify potentially eligible studies from inception to May 2020. Computer searches will be carried out using the Medical Subject Heading and keywords. There will be no limitations of language and publication status. The bibliographies of identified studies and review Articles will be manually screened to expand the number of eligible studies.

Main outcome(s): The primary outcomes are sensitivity and specificity. The secondary outcomes are positive likelihood ratio, negative likelihood ratio, and diagnostic odds ratio.

Quality assessment / Risk of bias analysis: The Quality Assessment of Diagnostic Accuracy Studies-2 (QUADAS-2) tool will be utilized to evaluate the risk of bias and methodological quality by two investigators independently.[21] Any discrepancies will be resolved via discussion with a senior author. The quality of each included study will be evaluated by an appraisal of the risk of bias of four domains and clinical applicability of three domains of the study characteristics. Four domains consisted of patient selection, index test, reference standard and flow and timing. Each domain will be evaluated for risk of bias, and the first three domains will be evaluated for applicability. The processing of the quality assessment will be performed utilizing RevMan 5.3 software (Nordic Cochrane Centre, Copenhagen, Denmark).

Strategy of data synthesis: The present meta-analysis will be conducted by Stata 12.0 (Stata Corporation, College Station, Texas). All statistical analyses will be performed by one investigator, who has experience in performing meta-analysis. The summary estimates of sensitivity, specificity, positive likelihood ratio, negative likelihood ratio and diagnostic odds ratio with corresponding $95 \%$ confidence intervals will be calculated using a bivariate random effect model in the present analysis, which indicate the accuracy of CEUS in the diagnosis of SRMs. Meanwhile, the summary receiver operator curve will be constructed and the area under the curve (AUC) will be calculated.

Subgroup analysis: We will operate subgroup analysis based on different study or patient characteristics, comparators, and outcomes.

Sensibility analysis: We will plan to conduct a sensitivity analysis by removing low quality studies to check the robustness of outcome results.

\section{Country(ies) involved: China.}

Keywords: contrast-enhanced ultrasound, small renal masses; meta-analysis; systematic review; diagnostic accuracy.

Contributions of each author:

Author 1 - Jiangfeng Wu.

Author 2 - Chao Wu.

Author 3 - Yunlai Wang.

Author 4 - Zhengping Wang. 